\title{
Prototype Development of a Shape Presentation System Using Linear Actuators
}

\author{
Tatsuo Motoyoshi ${ }^{(凶)}$, Sota Mizushima, Kei Sawai, Takumi Tamamoto, \\ Hiroyuki Masuta, Ken'ichi Koyanagi, and Toru Oshima \\ Department of Intelligent Systems Design Engineering, \\ Toyama Prefectural University, Toyama 939-0398, Japan \\ motoyosh@pu-toyama.ac.jp
}

\begin{abstract}
We here propose a three-dimensional shape presentation system using linear actuators which can control the displacement of a rod to a precision of $0.1 \mathrm{~mm}$. We assume that this system will be used as a device to present topographical/shape information to people with visual impairments. For this study, we produced a prototype system equipped with precision linear actuators, and conducted experiments to evaluate the capabilities of the system.
\end{abstract}

\section{Introduction}

When people with visual impairments access topographical/shape information, the information needs to be expressed tactilely. We previously proposed a computer-aided system enabling people with visual impairments to create and recognize their own figures by using a matrix braille display [1]. However, since a matrix braille display can indicate only the outline of the shape, it is unsuitable for indicating height and inclination information. Using figures created by a three-dimensional (3D) printer is expensive because all the information is needed to create the figures. We are therefore developing a 3D shape presentation system using linear actuators which can control the height of a rod. Since, the proposed system can show information about height and incline of topography precisely, it is useful for understanding a hazard map or a floor map for visual impairments.

\section{Shape Presentation System}

We previously produced a prototype of a 3D shape presentation system using linear actuators which we call THK:PCS-9RD [2] to indicate height and inclination information. The rod of each actuator can be moved to a maximum height of $50 \mathrm{~mm}$ and adjusted in increments of $1.25 \mu \mathrm{m}$.

The system consists of two linear actuators equipped with two finger plates, one operation handle, and one pen tablet. An operator holds the operation handle and sets two of his/her fingers on the finger plates, which are joined to the

(C) The Author(s) 2018

K. Miesenberger and G. Kouroupetroglou (Eds.): ICCHP 2018, LNCS 10897, pp. 226-230, 2018.

https://doi.org/10.1007/978-3-319-94274-2_31 
rods of the actuators, and then adjusts the handle position extensively while detecting the shape information. The coordinate position of the operation handle is detected by the pen tablet installed under the handle. The operation handle can be moved $135 \mathrm{~mm}$ vertically and $216 \mathrm{~mm}$ laterally. The system can control the height of each rod in increments of $0.1 \mathrm{~mm}$ in order to conform to the shape information. Each actuator communicates with a control computer through a CAN communication system (shown The configuration of proposed system is shown in Fig. 1).

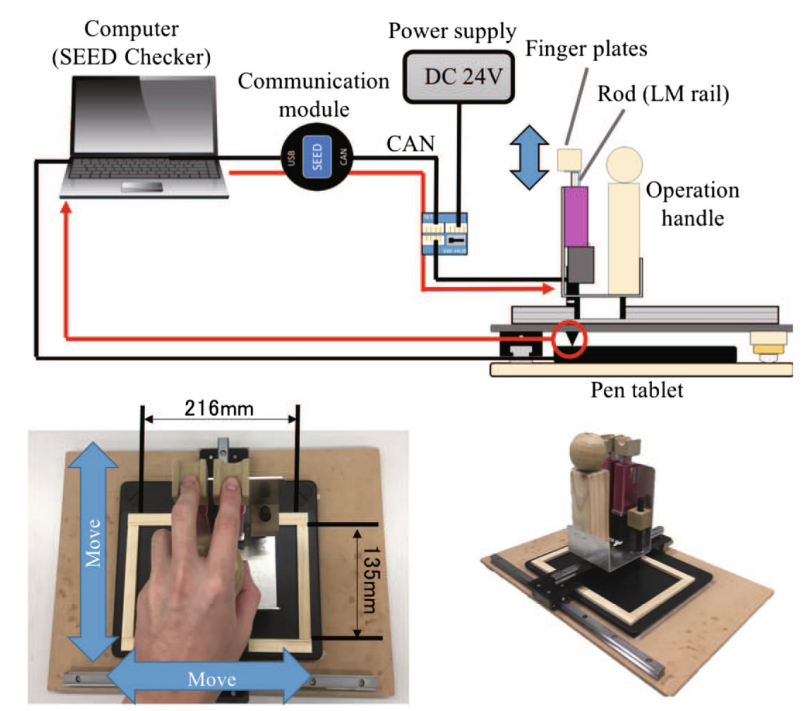

Fig. 1. Prototype of the shape presentation system

\section{Evaluation of the Prototype}

We conducted two experiments to evaluate the presentation capabilities of the prototype.

Exp. 1: We prepared information of four simple 3D objects representing square buildings. We recorded the time required to report the height differences between the objects, and calculated the accuracies of the recorded height orders.

Exp. 2: We prepared street map information for the proposed system and a tactile map. We recorded the times required to report street map information in each case.

\subsection{Exp.1}

For exp. 1, we recruited three people with normal sight as participants. Each participant wore a blindfold over his/her face eyes and was presented with a map 
on which four square buildings of differing heights were represented. We recorded the time required to report the layout and height order of the buildings. In proper order, buildings differed in height by $1 \mathrm{~mm}$. The map is shown in Fig. 2.

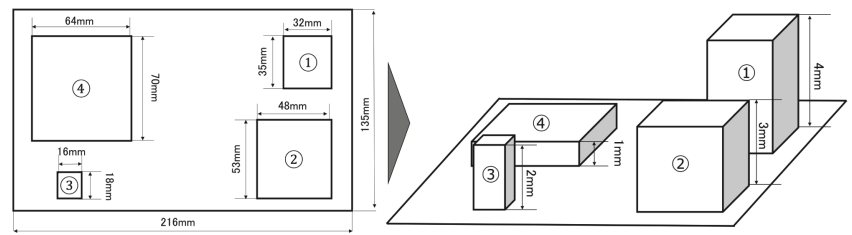

Fig. 2. Map for exp. 1

After the experiment, subjects were instructed to give the layout and height order of the buildings. Table 1 shows time required and results for each of the three participants.

Although participants A and B completed the task faster than did participant C, participants A and B failed to detect building (3), whereas participant $\mathrm{C}$ could detect all the buildings on the map. All participants could discriminate the height order of the buildings which they detected.

Table 1. Results

\begin{tabular}{l|l|l|l}
\hline Participant & Time & Height order & Number of recognized buildings \\
\hline A & $191 \mathrm{~s}$ & $\checkmark$ & 3 \\
\hline B & $476 \mathrm{~s}$ & $\checkmark$ & 3 \\
\hline C & $624 \mathrm{~s}$ & $\checkmark$ & 4 \\
\hline
\end{tabular}

\subsection{Exp. 2}

For exp. 2, we again recruited three people with normal sight as participants. Each participant wore a blindfold over his/her face eyes and was presented one at a time with the street maps shown in Fig. 3. Map 1 is for the proposed system and map 2 is the tactile map. In the experiment, each participant was told to detect the start and goal positions and structure of the street pattern on the map using either map 1 or 2 in the first session and the other map in the second session. Maps 1 and 2 have the same numbers of buildings and street corners. We recorded the time needed to detect the required information from the map. After the experiment, participants were asked to describe the route to the goal and the overall view of street pattern on the map.

When using the tactile map, all participants could detect the route to the goal faster than when using the proposed system. However, only participant $\mathrm{C}$ described the overall street pattern of the map correctly in this case. When 

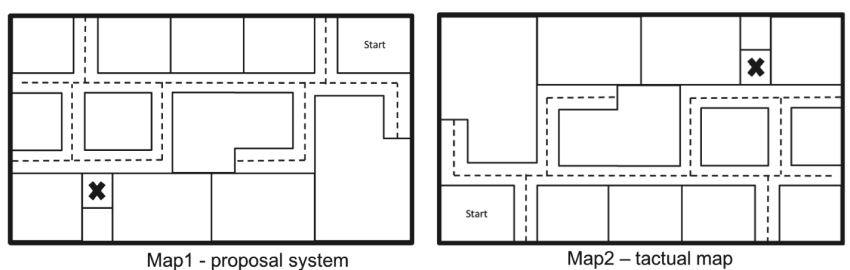

Fig. 3. Map for exp.2

using the proposed system, participants could only describe the route to the goal. All subjects commented that since they use only one hand in operating the proposed system, they found it difficult to understand the reference position. On the other hand, they found it easy to trace their route along the streets by using the proposed system.

Table 2. Results

\begin{tabular}{l|l|l|l|l}
\hline Participant & $\begin{array}{l}\text { Tactile map } \\
\text { time }\end{array}$ & Street pattern & $\begin{array}{l}\text { Proposed system } \\
\text { time }\end{array}$ & Street pattern \\
\hline A & $174 \mathrm{~s}$ & - & $190 \mathrm{~s}$ & - \\
\hline B & $590 \mathrm{~s}$ & - & $660 \mathrm{~s}$ & - \\
\hline $\mathrm{C}$ & $325 \mathrm{~s}$ & - & $1008 \mathrm{~s}$ & $\checkmark$ \\
\hline
\end{tabular}

\section{Summary}

We developed a 3D shape presentation system using linear actuators for people with visual impairments. We then conducted experiments to evaluate the presentation capability of a prototype. From the experimental results, we conclude that the proposed system can communicate height difference information at a $1 \mathrm{~mm}$ precision and is advantageous for tracing a streaked or striated pattern, such as a street pattern. In future work, we will add actuators for four fingers and a mechanism for a second hand to allow pointing at a reference position.

\section{References}

1. Takagi, N., Morii, S., Motoyoshi, T.: Prototype development of image editing systems available for visually impaired people and consideration of their user interfaces. J. Adv. Comput. Intell. Intell. Inform. (JACIII) 20(6), 961-967 (2016)

2. THK: Seed Picsel. http://seed-solutions.net/?qeng/node/29 
Open Access This chapter is licensed under the terms of the Creative Commons Attribution 4.0 International License (http://creativecommons.org/licenses/by/4.0/), which permits use, sharing, adaptation, distribution and reproduction in any medium or format, as long as you give appropriate credit to the original author(s) and the source, provide a link to the Creative Commons license and indicate if changes were made.

The images or other third party material in this chapter are included in the chapter's Creative Commons license, unless indicated otherwise in a credit line to the material. If material is not included in the chapter's Creative Commons license and your intended use is not permitted by statutory regulation or exceeds the permitted use, you will need to obtain permission directly from the copyright holder.

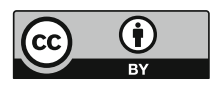

